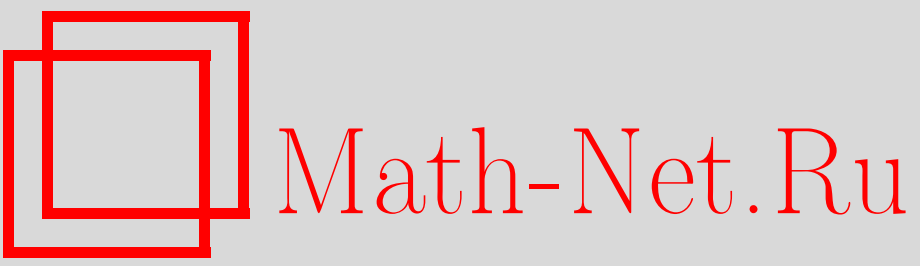

А. Ю. Плахов, Задача Ньютона о теле наименьшего сопротивления с ограниченным числом соударений, УМН, 2003, том 58, выпуск 1, 195-196

DOI: https://doi.org/10.4213/rm605

Использование Общероссийского математического портала Math-Net.Ru подразумевает, что вы прочитали и согласны с пользовательским соглашением

http://www . mathnet.ru/rus/agreement

Параметры загрузки:

IP : 52.6 .47 .48

26 апреля 2023 г., 12:50:48 


\title{
ЗАДАЧА НЬЮТОНА О ТЕЛЕ НАИМЕНЬШЕГО СОПРОТИВЛЕНИЯ С ОГРАНИЧЕННЫМ ЧИСЛОМ СОУДАРЕНИЙ
}

\author{
А. Ю. ПлАхов
}

1. Пусть $\Omega \subset \mathbb{R}^{d}, d=2,3$; обозначим $(x, v) \mapsto\left(x_{\Omega}^{+}(x, v), v_{\Omega}^{+}(x, v)\right)$ отображение рассеяния бильярдной динамической системы в $\mathbb{R}^{d} \backslash \Omega$ и рассмотрим интеграл

$$
R(\Omega)=\int_{L^{d}}\left(v_{\Omega}^{+}\left(x, v^{d}\right)-v^{d}\right) d x,
$$

где $v^{2}=(0,-1), v^{3}=(0,0,-1)$ и $L^{d}=\mathbb{R}^{d-1} \times\{0\}$. Вектор $R(\Omega)$ интерпретируется как сопротивление множества $\Omega$ однородному потоку точечных частиц, падающих вертикально вниз с единичной скоростью. Частицы движутся свободно вне множества $\Omega$ и отражаются по закону "угол падения равен углу отражения" в регулярных точках его границы.

Обозначим $\mathscr{B}_{d}(a, H)$ класс связных множеств $\Omega \subset \mathbb{R}^{d}$ таких, что (i) $B_{a} \times\{0\} \subset \Omega \subset B_{a} \times$ $[0, H]$, где $B_{a}=\left\{x \in \mathbb{R}^{d-1}:|x| \leqslant a\right\}$ - круг радиуса $a$ в $\mathbb{R}^{d-1}$, и (іi) интеграл (1) корректно определен, т.е. функция $v_{\Omega}^{+}\left(x, v^{d}\right)$ определена на подмножестве полной меры множества $L^{d}$ и измерима. Обозначим также $m(\Omega)$ максимально возможное число соударений с $\Omega$, которое испытьвает частица, падающая в направлении $v^{d}$.

Рассматривается задача минимизации величины сопротивления $|R(\Omega)|(1)$ в классе $\mathscr{B}_{d}(a, H)$, $d=2,3, a>0, H>0$. Кроме того, рассматривается следующая задача: определить наименьшее $m$ такое, что множество или семейство множеств, минимизирующие сопротивление, могут быть выбраны таким образом, что число соударений с ними не превосходит $m$.

Задача минимизации сопротивления рассматривалась И. Ньютоном для класса выпуклшх тел из $\mathscr{B}_{3}(a, H)$, обладающих вращательной симметрией относителњно координатной оси $O x_{3}$ [1]. В последние годы она изучалась для более широких классов тел из $\mathscr{B}_{3}(a, H)$, без предположений о выпуклости или симметрии, но всегда при условии, что каждая частица соударяется с телом не более одного раза, т.е. $m(\Omega)=1[2]-[5]$.

В настоящей работе показано, что

$$
\inf _{\Omega \in \mathscr{B}_{d}(a, H)}|R(\Omega)|=\rho_{d}(a, H),
$$

где $\rho_{2}(a, H)=2 a\left(1-H / \sqrt{a^{2}+H^{2}}\right), \rho_{3}(a, H)=0$, и построено семейство множеств $\Omega_{d}^{\varepsilon}(a, H)$, $\varepsilon>0$, минимизирующее сопротивление, $\lim _{\varepsilon \rightarrow 0}\left|R\left(\Omega_{d}^{\varepsilon}(a, H)\right)\right|=\rho_{d}(a, H)$. При $d=2$ и при $d=3, H \geqslant a / 2$ наибольшее число соударений для множеств, входящих в семейство, равно 2 , $m\left(\Omega_{d}^{\varepsilon}(a, H)\right)=2$, и это значение нельзя улучшить, т.е. нельзя предъявить минимизирующее множество или семейство множеств с числом соударений, не превосходящим 1 . При $d=3$ и $0<H<a / 2$ имеем $m\left(\Omega_{3}^{\varepsilon}(a, H)\right)=3$. Наша гипотеза состоит в следующем: существует число $0<\kappa \leqslant 1 / 2$ такое, что при $H \geqslant \kappa a$ найдется минимизирующее семейство множеств, для которых число соударений не превосходит 2 , а при $0<H<\kappa a$ такого семейства не существует.

2. Пусть $d=2$. Обозначим $\widehat{\Omega}$ вьпуклую оболочку множества $\Omega \in \mathscr{B}_{2}(a, H)$. Часть границы $\widehat{\Omega}$, обращенная вверх, является графиком выпуклой вверх функции $f:(-a, a) \mapsto(0, H]$. Обозначим А множество точек графока $f$, принадлежащих $\partial \Omega$, и $\mathrm{B}=\bigcup_{i} \mathrm{~B}_{i}$ множество остальных точек граф̆ика, $\mathrm{B}_{i}$ - его связные компоненты. Если падающая частица в первый раз пересечет $\partial \widehat{\Omega}$ в точке $\left(x_{1}, f\left(x_{1}\right)\right) \in \mathrm{B}_{i}$, то через некоторое время она вновь пересечет $\mathrm{B}_{i}$ в обратном направлении и далее будет двигаться свободно со скоростью $v^{+}\left(x, v^{2}\right)$, образующей с осью $O x_{2}$ больший угол, чем касательная к графику $f$ в соответствующей точке. Отсюда следует неравенство для второй компоненты скорости: $v_{2}^{+}\left(x, v^{2}\right)>-\left|f^{\prime}\left(x_{1}\right)\right| / \sqrt{1+f^{\prime 2}\left(x_{1}\right)}$, где $x=\left(x_{1}, 0\right)$. То же неравенство справедливо и для частицы, отразившейся в точке из А. Следовательно,

$$
|R(\Omega)|>\int_{-a}^{a}\left(1-\left|f^{\prime}(\xi)\right| / \sqrt{1+f^{\prime 2}(\xi)}\right) d \xi ;
$$

последний интеграл достигает своего минимума $\rho_{2}(a, H)$ на функции $f^{*}(\xi)=H(1-|\xi| / a)$. 
Остается построить семейство множеств из $\mathscr{B}_{2}(a, H)$, сопротивление которых сходится к $\rho_{2}(a, H)$. Пусть $0 \leqslant \alpha \leqslant a-\varepsilon^{2}, \gamma=\alpha+\varepsilon^{3}, \beta=\alpha+\varepsilon^{2} ;$ обозначим $p$ параболу с вершиной $\left(\gamma, f^{*}(\gamma)-\varepsilon\right)$, фокусом $F=\left(\gamma, f^{*}(\gamma)-\varepsilon^{3}\right)$, и обозначим $E_{1}=\left(\beta, f^{*}(\beta)\right)$ и $E_{2}$ точку пересечения $p$ с прямой $x_{1}=\beta$. Проведем прямую $l$ через точку $F$ таким образом, чтобы отрезки $E_{1} F$ и $E_{2} F$ находились по одну сторону от $l$ и образовьвали с ней равные углы. Пусть $f^{*}(\gamma)-\varepsilon>0$; обозначим $K_{\alpha, \varepsilon}$ открытое множество точек $\left(x_{1}, x_{2}\right), \alpha<x_{1}<\beta$, лежащих выше параболы $p$ и ниже прямой $l$ и графиика $f^{*}$. Если же $f^{*}(\gamma)-\varepsilon \leqslant 0$, то положим $K_{\alpha, \varepsilon}=\varnothing$. Множество, симметричное $K_{\alpha, \varepsilon}$ относительно оси $O x_{2}$, обозначим $K_{-\beta, \varepsilon}$. Положим $\varepsilon=\sqrt{a / n}$ и определим $\Omega_{2}^{\varepsilon}(a, H)=\left\{\left|x_{1}\right| \leqslant a, 0 \leqslant x_{2} \leqslant f^{*}\left(x_{1}\right)\right\} \backslash\left(\bigcup_{k=-n}^{n-1} K_{k \varepsilon^{2}, \varepsilon}\right)$. Для любого $x_{1} \in[-a, a]$, за исключением множества меры $O(\varepsilon)$, соответствующая частица, падающая на $\Omega_{2}^{\varepsilon}(a, H)$, попадает в одно из множеств $K_{k \varepsilon^{2}, \varepsilon}$, испытывает соударения в точке из $p$ и в точке из $l$ и затем движется свободно, причем вторая компонента ее скорости равна $v_{2}^{+}=-H / \sqrt{a^{2}+H^{2}}+O(\varepsilon)$. Отсюда следует, что $\left|R\left(\Omega_{2}^{\varepsilon}(a, H)\right)\right|=\rho_{2}(a, H)+O(\varepsilon)$, что и требовалось.

3. Перейдем к трехмерному случаю. Сначала проведем построения в плоскости $\mathbb{R}_{x_{1} x_{3}}^{2}$; ось $O x_{1}$ считаем горизонтальной, а ось $O x_{3}$ - вертикальной. Рассмотрим параболу $\Gamma_{\varepsilon}$ с вертикальной осью, вершиной $(a-\varepsilon, 0)$ и фокусом $F_{\varepsilon}=\left(a-\varepsilon, \frac{a-\varepsilon}{2}\right)$. Обозначим $C_{\varepsilon}$ множество точек $\left(x_{1}, x_{3}\right), 0 \leqslant x_{1} \leqslant a, x_{3} \geqslant 0$, лежащих ниже параболы $\Gamma_{\varepsilon}$ или на ней. Далее, обозначим $B_{\varepsilon}$ точку пересечения $\Gamma_{\varepsilon}$ с прямой $x_{1}=a$ и обозначим $M_{\varepsilon}$ участок параболы $\Gamma_{\varepsilon}^{\prime}$ с фокусом $F_{\varepsilon}$ и осью $F_{\varepsilon} B_{\varepsilon}$, содержащийся в множестве $x_{1} \geqslant a-\varepsilon, x_{3} \geqslant \frac{a-\varepsilon}{2}$, причем $\Gamma_{\varepsilon}^{\prime}$ выбираем так, чтобы $M_{\varepsilon}$ целиком принадлежал множеству $x_{1} \leqslant a, x_{3} \leqslant a / 2$. Определим $\Omega_{3}^{\varepsilon}(a, H), H \geqslant a / 2$, как объединение плоского множества $x_{1}=0,\left|x_{2}\right| \leqslant a, 0 \leqslant x_{3} \leqslant a / 2$ и множества $\mathscr{Q}\left(C_{\varepsilon} \cup M_{\varepsilon}\right)$, полученного в результате вращения $C_{\varepsilon} \cup M_{\varepsilon}$ вокруг оси $O x_{3}$. Частица, отразившаяся от $\mathscr{Q}\left(C_{\varepsilon}\right)$ и затем от $\mathscr{Q}\left(M_{\varepsilon}\right)$, далее движется свободно со скоростью, образующей с $v^{3}$ угол порядка $\varepsilon$. Таким образом, $v^{+}\left(x, v^{3}\right)-v^{3}=O(\varepsilon)$ для всех значений $x \in L^{3}$, за исключением множества плоской меры $O(\varepsilon)$, следовательно, $\left|R\left(\Omega_{3}^{\varepsilon}(a, H)\right)\right|=O(\varepsilon)$.

Предположим теперь, что $H<a / 2$. В плоскости $\mathbb{R}_{x_{1} x_{3}}^{2}$ рассмотрим параболу $G_{\varepsilon}$ с вершиной $(\varepsilon, 0)$, вертикальной осью и фокусом $P_{\varepsilon}=(\varepsilon, b-2 \varepsilon)$ и параболу $G_{\varepsilon}^{\prime}$ с вершиной $(0, b-2 \varepsilon)$, фокусом $P_{\varepsilon}$ и горизонтальной осью. Назовем блоком $B_{b, \varepsilon}$ множество точек $\left(x_{1}, x_{3}\right)$, $0 \leqslant x_{1} \leqslant b,-\infty<x_{3} \leqslant b$, лежащих либо ниже параболы $G_{\varepsilon}$ (или на ней), либо выше верхней ветви параболы $G_{\varepsilon}^{\prime}$. Множество $C_{b, \varepsilon, n}=\bigcup_{k=0}^{n-1}\left(u_{k}+B_{b, \varepsilon}\right)$ есть объединение $n$ экземпляров блока, сдвинутых на векторы $u_{k}=(b k, 2 \varepsilon(n-1-k))$; соседние экземпляры примькают друг к другу по боковым сторонам, с разницей по высоте $2 \varepsilon$. Наконец, обозначим $J_{b, \varepsilon, n}$ отрезок с концами $(b n, b-2 \varepsilon)$ и $K=(\zeta, b+2 \varepsilon(n-1)) ; \zeta<b n$ выбирается таким образом, чтобы полученньй отрезок $J_{b, \varepsilon, n}$ служил биссектрисой угла $K^{\prime} K K^{\prime \prime}$, где $K^{\prime}=(b n, b / 2)$, $K^{\prime \prime}=(b n, b+2 \varepsilon(n-1))$. Выберем $b$ и $n$ таким образом, чтобы $b<H / 2, b n=a$, и определим $\Omega_{3}^{\varepsilon}(a, H), H<a / 2$, как объединение плоского множества $x_{1}=0,\left|x_{2}\right| \leqslant a$, $0 \leqslant x_{3} \leqslant H$ и множества $\mathscr{Q}\left(C_{b, \varepsilon, n} \cup J_{b, \varepsilon, n}\right) \cap\left\{x_{3} \geqslant 0\right\}$. Для всех значений $x \in L^{3},|x| \leqslant a$, за исключением множества плоской меры $O(\varepsilon)$, соответствующая частица дважды отразится в точках $\mathscr{Q}\left(C_{b, \varepsilon, n}\right)$, затем отразится в точке $\mathscr{Q}\left(J_{b, \varepsilon, n}\right)$ и далее будет двигаться свободно со скоростью $v^{+}\left(x, v^{3}\right)=v^{3}+O(\varepsilon)$. Следовательно, и в этом случае $\left|R\left(\Omega_{3}^{\varepsilon}(a, H)\right)\right|=O(\varepsilon)$.

\section{СПИСОК ЛИТЕРАТУРЫ}

[1] I. Newton. Philosophiae naturalis principia mathematica, 1686. Рус. пер.: И. Ньютон. Классические начала натуралшной философии. М.: Наука, 1989. [2] G. Buttazzo, В. Каwohl // Math. Intelligencer 1993. V. 15. № 4. P. 7-12. [3] G. Buttazzo, V. Ferone, B. Kawohl // Math. Nachr. 1995. V. 173. P. 71-89. [4] F. Brock, V. Ferone, B. Kawohl // Calc. Var. Partial Differential Equations. 1996. V. 4. №6. P. 593-599. [5] T. Lachand-Robert, M. A. Peletier // Math. Nachr. 2001. V. 226. P. 153-176. 